\title{
Embedded System for Automatic Traffic Violation Monitoring and Alerting
}

\author{
V. Ramya \\ Assistant Professor, Dept. of \\ CSE, Annamalai University, \\ Chidambaram.
}

\author{
B. Palaniappan \\ Dean, FEAT, Head, Dept. of \\ CSE, Annamalai University, \\ Chidambaram.
}

\author{
M. Aruljothi \\ PG Scholar, \\ Dept. of CSE, Annamalai \\ University, Chidambaram.
}

\begin{abstract}
Safety and comfort of road users is becoming a matter of grave concern. It is essential to build a safer and much more reliable system for traffic control and management, since the number of on road accidents has shoot up greatly with the increase in vehicle traffic $[1,10]$. The objective of this project is to introduce a system which detects all kind of violations at a street intersection such as speed violation, stop line violation and lane violation during red light running and thereby we can trace each and every individual vehicle. Two rows of IR transmitters are used for detecting the violations. One is to warn the driver, whenever he crosses the first stop line during red signal, by switching on the alarm circuit which is fixed in the vehicle, and the alarm is a continuous one until he stops the vehicle. Second one is to transmit the details of the vehicle to the RTO office, if he cross the second stop line and it will ready to turn off the ignition whenever he tries to stop the vehicle. Once the vehicle is turned off, it will reset only through the Road Traffic Officer (RTO). The proposed system has two sections one is the receiver which is fixed in the vehicle and the second one is the transmitter which is fixed in the RTO office. Moreover, the details of the vehicle is monitored and transmitted to the RTO section and the details of the vehicle are displayed in the RTO section whenever the vehicle violates the traffic rule.
\end{abstract}

\section{Keywords}

Embedded System, Intelligent vehicle, Microcontroller RF communication, Sensors.

\section{INTRODUCTION}

Now a day's embedded system is a part of our life and will be even more prevalent in the future. Most of them perform simple control and support tasks on command in a preprogrammed manner, but that is not the end of the line. Increasingly they are designed to carry out autonomous tasks including "intelligence" (Smart Systems) they are also designed to be able to decide, based on complex inputs and situation awareness that is, what to do under circumstances defined by an unreliable environment. Many security systems that start from security lock codes to finger print scanner, iris scanner for security locks are the result of development in the field of embedded systems [2, 7]. Many safety systems such as speed control in school vans, fire detection system and smoke detection systems in apartments, laboratory, factories etc., and intruder detection in protected areas are the major embedded systems that are used widely around the world. Hence, these types of embedded systems play a major role in safety and security systems. Designing autonomous vehicles are the next step in this direction of development in the area of embedded technology.

\section{SYSTEM DESIGN}

The system design process consists of three sections. They are Traffic signal section, Vehicle section, RTO (Regional Transport Office) section.

\subsection{Traffic Signal Section}

Fig 1 shows the block diagram of the Traffic signal Section. This module comprises of one AT89C51 Micro controller and two LEDs for Red and Green signal. This section requires only less number of ports and hence AT89C51 Microcontroller is sufficient.

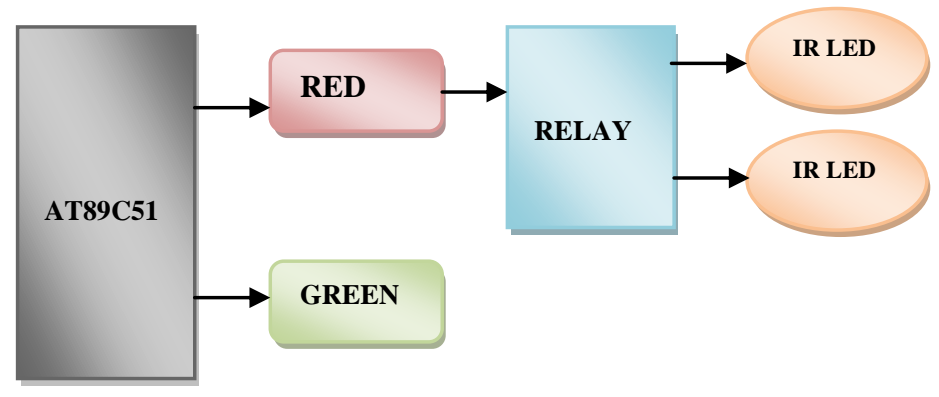

Fig 1: Traffic Signal Section

\subsection{Vehicle Section}

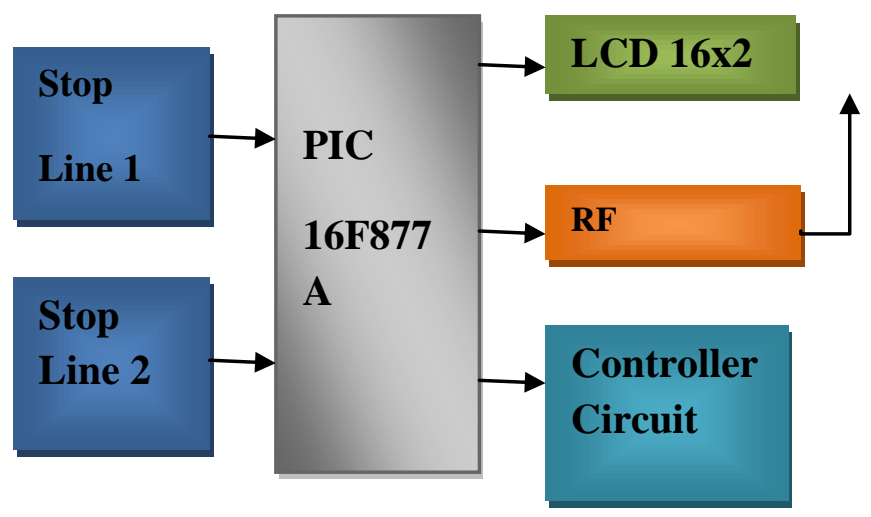

Fig 2: Vehicle Section 
Fig 2 shows the block diagram of the Vehicle section module. It includes PIC16F877A micro controller to control the traffic violation and RF module for transmission and reception purpose. LCD display is interfaced with microcontroller which displays the violation information at the vehicle side.

\subsection{RTO Section}

Fig 3 shows the block diagram of the RTO section module. This section comprises of PIC16F877 microcontroller, reset circuit to reset the vehicle and RF transmitter for transmitting the message to the vehicle.

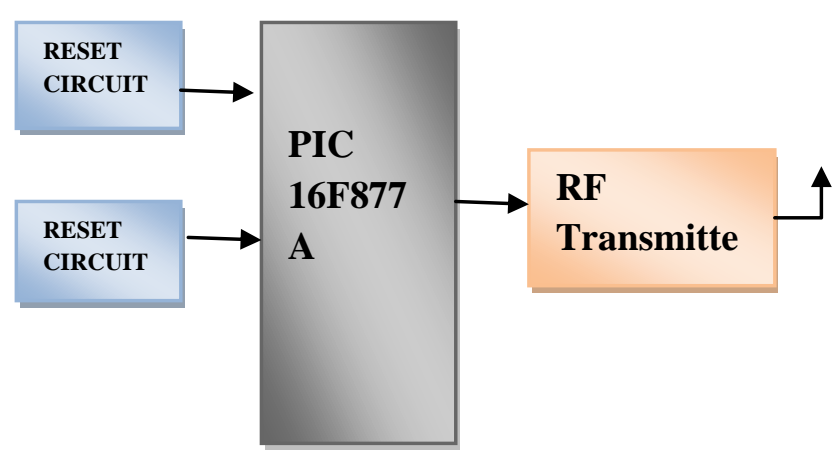

Fig 3: Rto Section

\section{HARDWARE DESCRIPTION}

The proposed system mainly aims to reduce the accident that happened due to traffic violation and also to provide safety to the human lives and vehicles in the accident prone zone. Hence, special equipment is added to the vehicle to provide this safety measure.

\subsection{Microcontroller \\ 3.1.1.PIC16F877}

Microcontrollers are used to process information or to control some devices. PIC16F877A have 40 pin and internal FLASH memory technology and it can be write and erase until thousand times. PIC16F877A perfectly fits for many applications ranging from automotive industries, controlling home appliances, industrial instruments, remote sensors, electrical door locks, and robotics and safety devices. It is also ideal for smart cards as well as for battery supplied devices because of its low consumption. EEPROM memory makes it easier to apply microcontrollers to devices where permanent storage of various parameters is needed. The low cost, low space and power consumption, easy handling and flexibility makes the PIC16F877A applicable to even in many areas where microcontrollers had not previously been considered (example: timer functions, interface replacement in larger systems, coprocessor applications, etc.). The pin diagram of PIC16F877 is shown in fig 4.

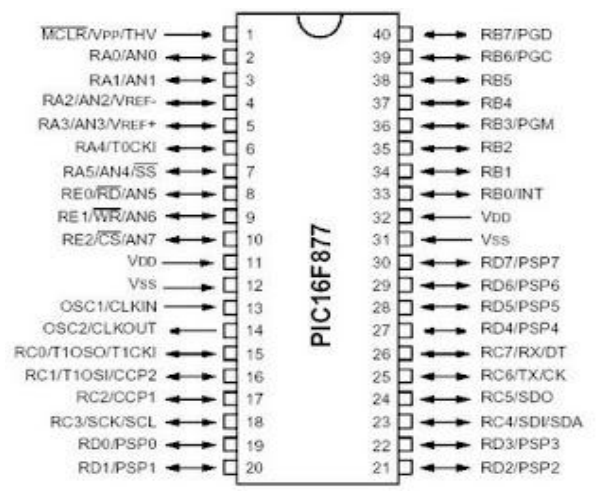

Fig 4: Pin Diagram of Pic16f877

\subsubsection{ATMEL89C51}

The AT89C51 is a low-power, high-performance CMOS 8-bit microcomputer with 4 Kbytes of Flash Programmable and Erasable Read Only Memory (EPROM). The on-chip Flash allows the program memory to be reprogrammed in-system or by a conventional non volatile memory programmer. By combining a versatile 8-bit CPU with Flash on a monolithic chip, this controller is a powerful microcomputer which provides a highly flexible and cost effective solution to many embedded control applications. It is provided with memory not only RAM but also ROM, parallel and serial port (Universal Asynchronous Receiver/Transmitter), etc which are all in one single chip. Flash EPROM is loaded with programs which include the instructions that will be compiled by the micro controller. Besides that, it can be reused for many times. In this work the AT89C51 microcontroller is used at the traffic light section.

\subsection{RF Communications}

The RF module operates at Radio Frequency and the corresponding frequency range varies between $30 \mathrm{kHz}$ to 300 GHz. Transmission through RF is better than IR (infrared) due to many reasons. Firstly, signals through RF travel through longer distances which make it suitable for long range applications. IR mostly operates in line-of-sight mode; RF signals can travel even when there is an obstruction between transmitter and receiver and moreover RF transmission is stronger and reliable than IR transmission. RF communication uses a specific frequency unlike IR signals which are affected by other IR emitting sources. This RF module comprises of an $\mathrm{RF}$ transmitter and an $\mathrm{RF}$ receiver $(\mathrm{Tx} / \mathrm{Rx})$ and this pair operates at a frequency of $434 \mathrm{MHz}$. The transmission occurs at the rate of $1 \mathrm{Kbps}-10 \mathrm{Kbps}$. The transmitted data is received by an RF receiver operating at the same frequency as that of the transmitter $[3,11]$.

\subsubsection{RF Transmitter}

The RF transmitter is a 4-pin chip that acts as antenna to transmit the signals at fixed frequencies. The RF transmitter is shown in figure 5 . This chip together with the encoder HT12$\mathrm{E}$ acts as a transmitter so as the signals can be decoded only at the code match at the receiver side. This transmission ranges up to 20 meters that can be either increased or decreased by the frequency of the signal that we are transmitting. The RF transmitter has a ground pin and input voltage of $5 \mathrm{~V}$, the 
output pin is the antenna pin through which the encoded data from HT12-E are transmitted [3, 12]. Interfacing of RF transmitter with PIC16f877 is shown in figure 6.

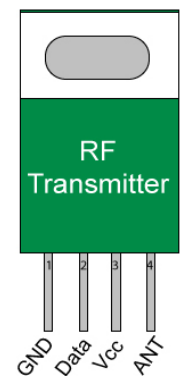

Fig 5: Rf Transmitter

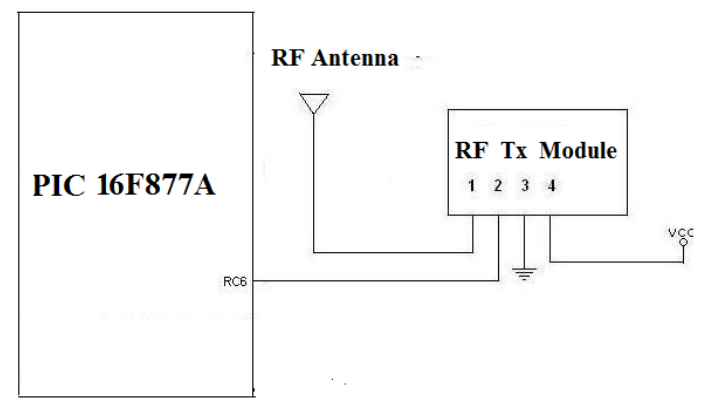

Fig 6: Interfacing Of Rf Transmitter With Pic16f877

\subsubsection{RF Receiver}

A tuned radio frequency receiver composed of several tuned radio frequency amplifiers followed by circuits to detect and amplify the audio signal. The RF receiver is an 8-pin chip that has antenna to receive the signals transmitted by the RF receiver. This chip together with the decoder forms the receiver circuit so as to decode the signals from the transmitter and to receive only the required signals. In the same way the receiver pin has a ground pin and input voltage of $5 \mathrm{~V}$. The antenna pin acts as the input pin that receives the signal and then sends them to the decoder through serial data output pin [2,3]. Interfacing RF receiver with PIC $16 \mathrm{~F} 877$ is shown in figure 8 .

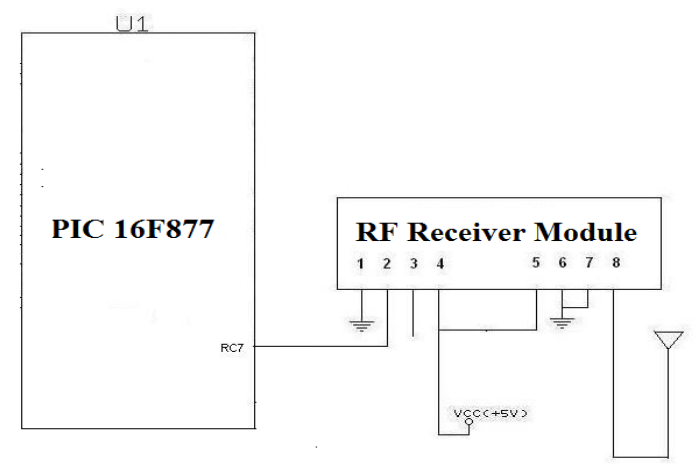

Fig 8: Interfacing Of Rf Receiver With Pic16f877

\subsection{LCD Display}

A liquid crystal display (LCD) is a thin, flat display device made up of any number of colour or monochrome pixels arrayed in front of a light source or reflector. It is often utilized in battery-powered electronic devices because it uses very small amounts of electric power. The most commonly used Character based LCDs are based on Hitachi's HD44780 controller or other which are compatible with HD44580.The most commonly used LCDs found in the market today are 1 Line, 2 Line or 4 Line LCDs which have only 1 controller and support at most of 80 characters, whereas LCDs supporting more than 80 characters make use of 2HD44780 controllers. Interfacing LCD display with PIC16F877 is shown in figure 10.

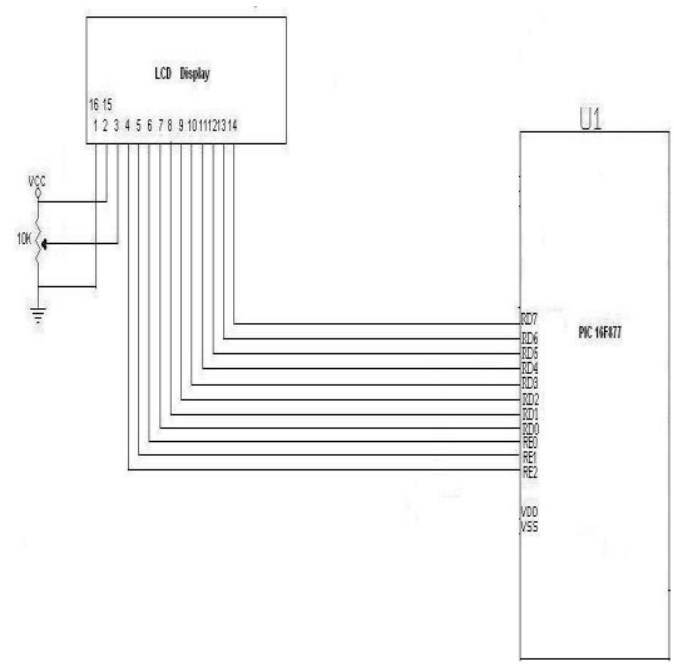

Fig 10: Interfacing Lcd Display With Pic16f877

Most LCDs with 1 controller has 14 Pins and LCDs with 2 controller has 16 Pins (two pins are extra in both for backlight LED connections).

Fig 7: Rf Receiver 


\subsection{Relay Circuit}

The Relay driver Circuit Works as follows and the output of the controller decides Relay operation. The Relay works only if the Positive and the Ground is connected to the appropriate terminals of the relay coil. The relay is controlled by controlling the ground signal given to the relay using a transistor. When the output of the controller is high, then the transistor conducts, allowing the low potential to reach one end of the relay, which results in switching of the relay form $\mathrm{NC}$ to NO. When the output is Low, the transistor will not conduct. The ground is not applied to the relay coil and hence the relay remains in NC position. Using this normally open (NO) and Normally Closed (NC) contacts we can switch any supply AC or DC. The supply to the operated device should be given to the Common of the relay and the output from the NO is given to the Device. So that when the relay is switched on the device gets supply and start working. The relay circuit is shown in figure 11 .

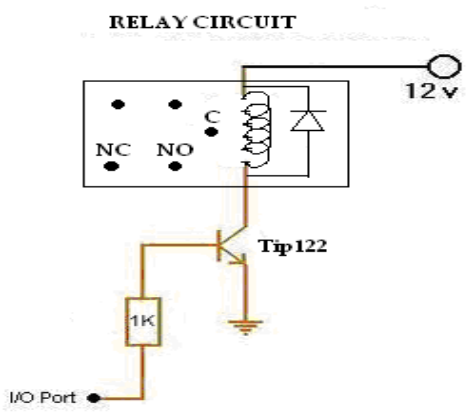

Fig 11: Relay Circuit

\subsection{IR Sensor}

The IR (Infra Red) sensor is basically an electronic device used to sense the surroundings by emitting and detecting the IR rays. The object detection principle using IR LED is shown in figure 12. Infrared sensors can measure and detects the heat of an object, as well as motion of an object. Many of these types of sensors only measure infrared radiation, else emitting the radiation. And these are known as Passive InfraRed (PIR) sensors. Any types of objects can emit some form of thermal radiation, usually in the infrared spectrum. Thus radiations are not visible to our eyes, but it can be detected by sensor. In a typical infrared sensor like motion detector, the radiations enter in to the front of the sensor and reach itself at the centre of the device. This part may be composed of more than one individual sensor, each of them being made from pyroelectric materials, whether natural or artificial. The pyroelectric materials generate an electrical voltage when it is heated or cooled. Object or radiation detection is the same principle in all Infra-Red proximity sensors [16]. An Infra red light is reflected when it hits in front of sensor through IR-LEDs. Interfacing IR sensors with PIC16F877 is shown in figure 13.

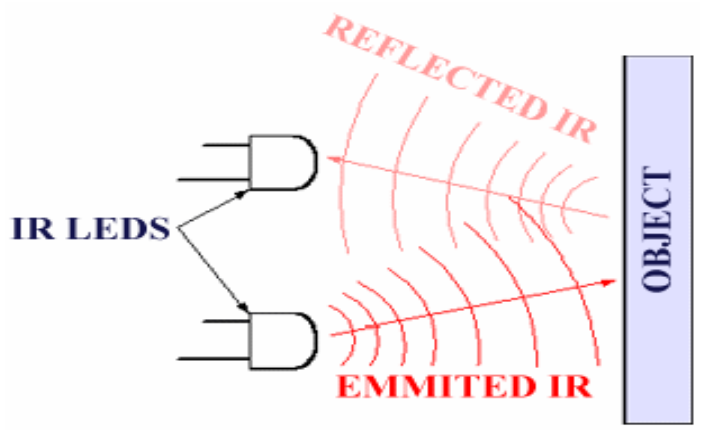

Fig 12: Object Detection Using Ir Led's

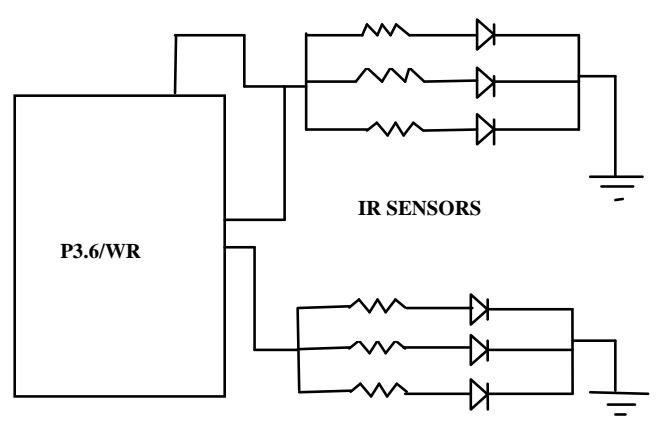

Fig 13: Interfacing Ir Sensor With The Pic16f877

\section{SOFTWARE DESCRIPTION 4.1. Embedded C}

The embedded system market is growing rapidly and hence there is an increasing demand to write application programs in a high-level language such as $\mathrm{C}$. The reasons behind this are, embedded system programming is more complex (and hence are difficult to maintain in assembly language), processors are needed to more frequent re-adaption of applications to new instruction sets and hence the lifespan of the processor model is decreased. To address these issues, the code re-usability is needed which is achieved with the help of C-level programming. As the $\mathrm{C}$ language has matured over the years, various extensions for accessing basic I/O hardware (iohw) registers have been added to address deficiencies in the language. Today almost all C compilers for embedded systems support some method of direct access to iohw registers from the $\mathrm{C}$ source level. Specific embedded-systems deficiencies in $\mathrm{C}$ have been addressed to reduce application dependence on assembly code. The Embedded c specification brings back the roots of $\mathrm{C}$ to embedded systems as primarily a high-level language means of accessing the processor. The main advantage of embedded $\mathrm{C}$ is it supports in-line assembly [3]. 


\subsection{Displaying Violation information}

\subsubsection{Violation information at Vehicle section}

During red light running in the signal, and if the vehicle crosses the first stop line the violation information "Stopline1 Violated" is displayed in the LCD which is fixed in the vehicle. The sample code for displaying the stop line1 violation information is given below.

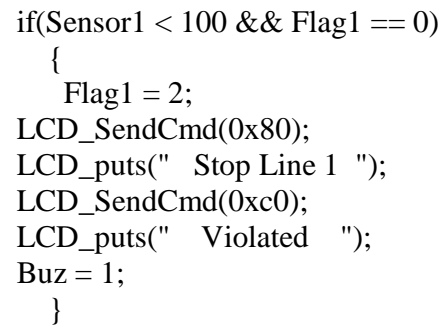

Suppose the vehicle cross the second stop line, the vehicle is halted, whenever the driver turned off the ignition and the halted information "Engine Halted Check RTO" is displayed in LCD fixed in the vehicle, and the corresponding code is given below.

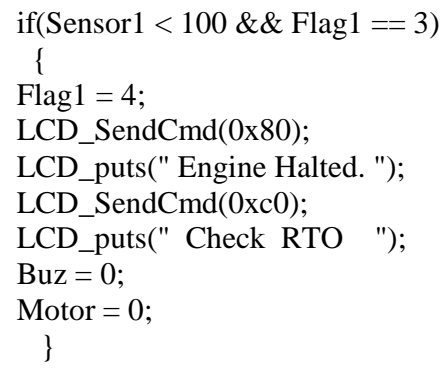

\subsubsection{Violation information at RTO section}

If the vehicle crosses the second stop line, the violation information "Violated Vehicle" is displayed in the LCD which is fixed in the RTO section:

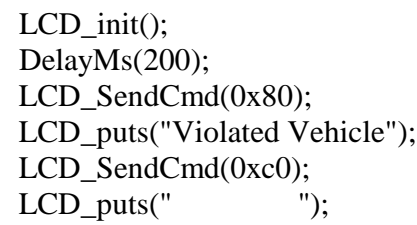

Simultaneously the details of the vehicle such as car number and the owners name is displayed in LCD fixed in the RTO module:

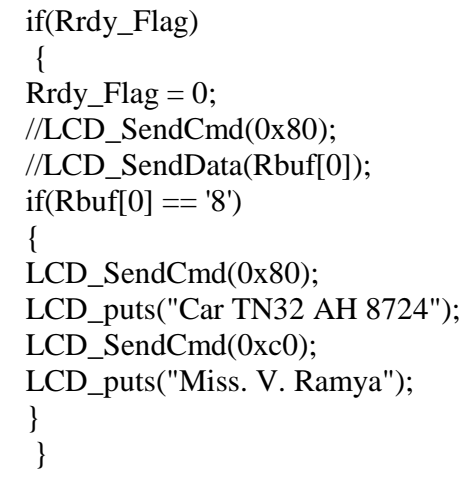

\section{IMPLEMENTATION}

The Fig 14 shows the hardware design of the traffic signal section. It comprises Transformer, Voltage regulator and LEDs. The Green and Red LEDs are running alternatively. If the Green light is glowing, the vehicle pass the stop lines freely without any alert but if the Red light is glowing the alarm is generated and also the alert message is displayed in the vehicle.

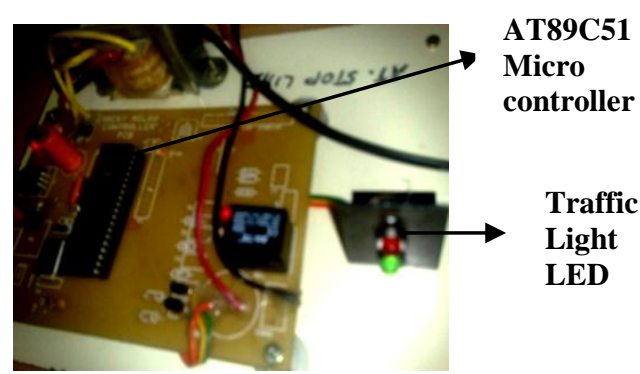

Fig 14: System Design Of The Traffic Signal Section

The Fig 15 shows the Hardware design of the Vehicle. An Alarm is fixed in the vehicle, which generates an alarm whenever the vehicle violates the first stop line and it will provide the warning message in the $\mathrm{LCD}$, whenever it violates the second stop line. The RTO section comprises a reset circuit which resets the vehicle whenever it violates the stop line. The RF module fixed in it, receives the message from the vehicle. An LCD display at this section displays the details of the vehicle; if it crosses the second stop line the reset button fixed in the RTO section only resets the vehicle. The system design of the RTO section is shown in the fig 16 .

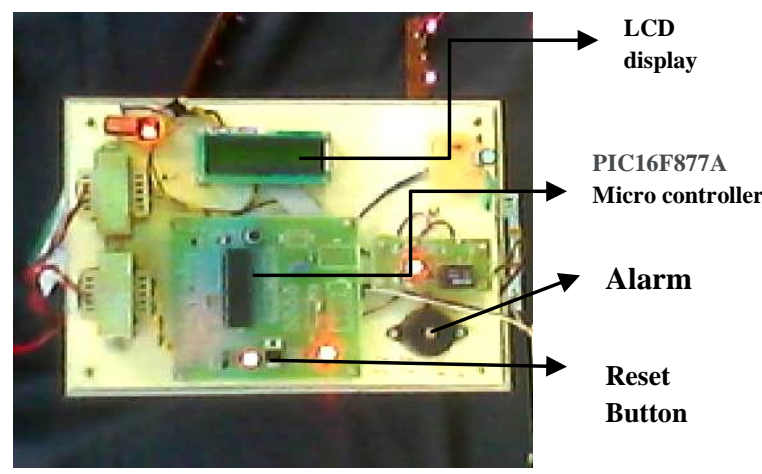

Fig 15: System Design of Thevehicle Section

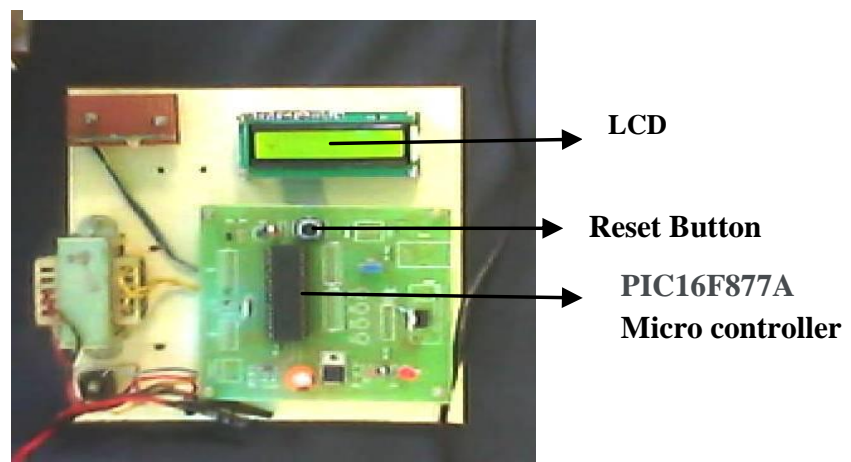

Fig 16: System Design of the Rto Section 
The Fig 17 shows the overall proposed system. It consists of three sections, one is Traffic signal section, second one is vehicle section and the third one is RTO section. During red light running in the signal, the sensor fixed in the two stop line matches with the sensors at the vehicle and then generates an alarm in the vehicle.

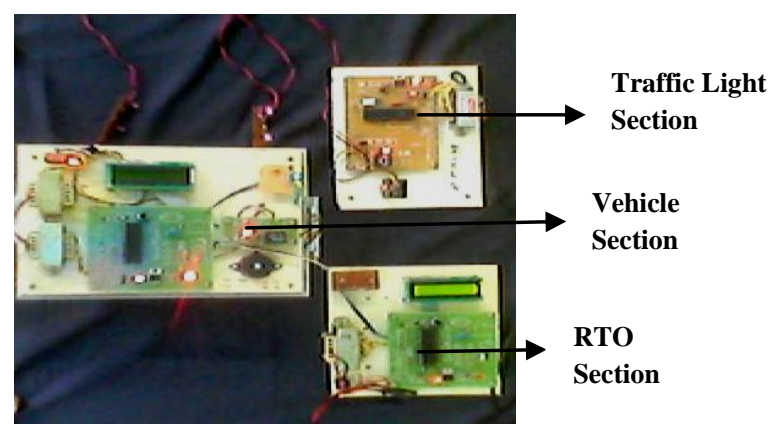

Fig 17: Prototype of the Proposed System

The Fig 18 displays the vehicle alert message in the LCD display which is fixed in the vehicle, if it crosses the first stop line.

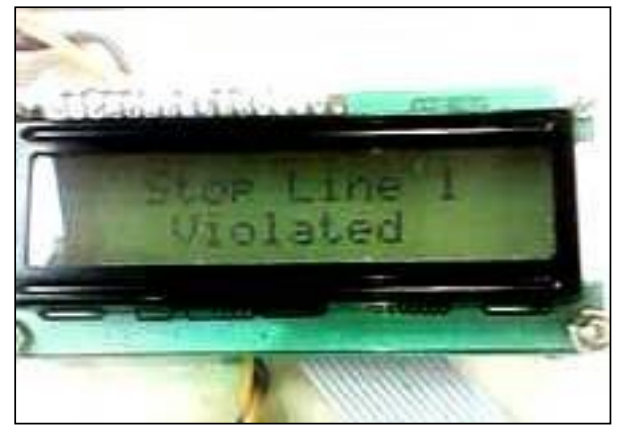

Fig 18: Vehicle Alert Message

The Fig 19 displays the vehicle alert message in the LCD display which is fixed in the RTO section, if the vehicle crosses the second stop line. The circuit diagrams of the three sections are shown in fig 16, 17 and 18.

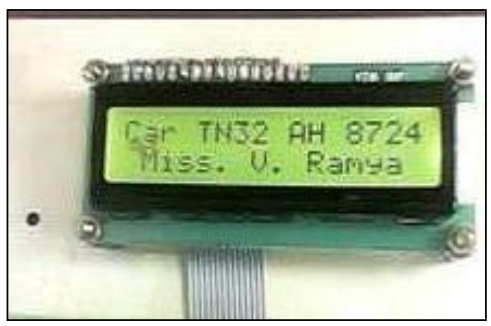

Fig 19: Vehicle Details

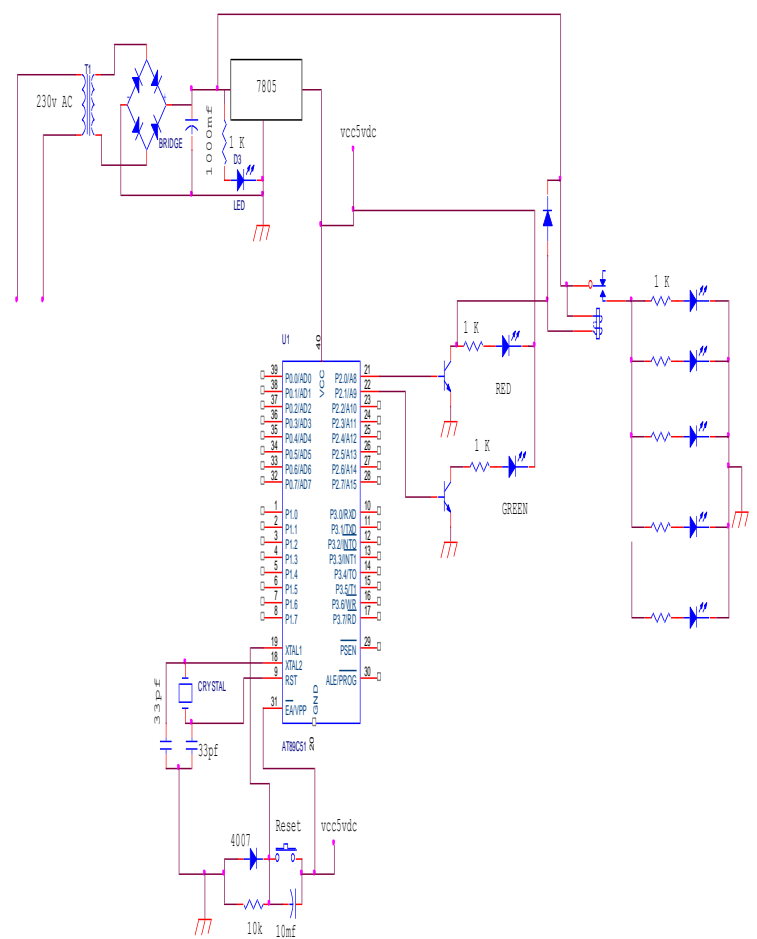

Fig 20: Circuit Diagram of the Signal Section

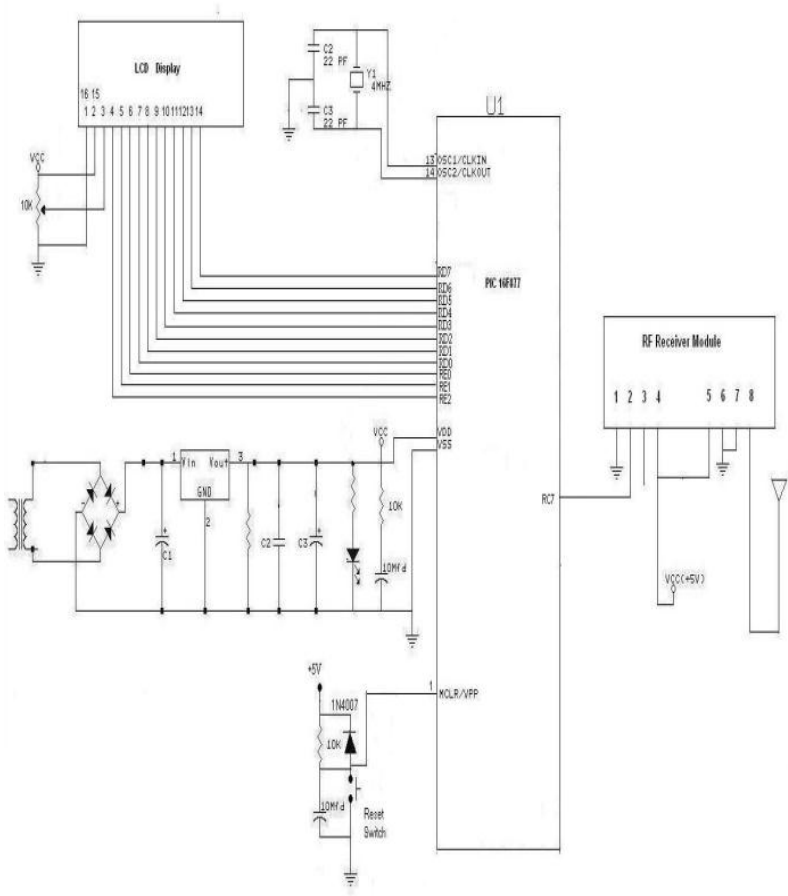

Fig 21: Circuit Diagram of the Rto Section 


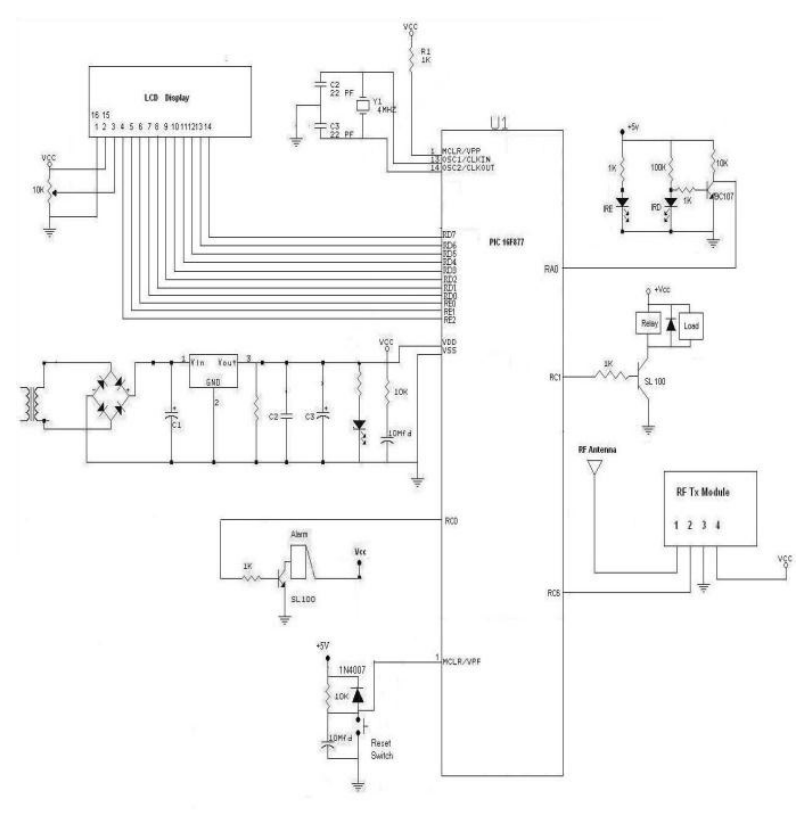

Fig 22: Circuit Diagram of the Vehicle Section

\section{CONCLUSION}

In this work, embedded system for automatic traffic violation monitoring, the traffic frequency is measured as a function of number of vehicles which comes under the contact of sensing unit. We have implemented a microcontroller-based system that automatically performs the functions of monitoring traffic violation using PIC16F877. This system is easy to install, strong function head and easy to promote and implement. In future we planned to extend this project in such a way that the system can monitor more number of vehicles and to send message about the traffic violation to the respective people like check post and police station through GSM.

\section{REFERENCES}

[1] V.Ramya, B.Palaniappan and K.Karthick, "Embedded controller for vehicle In-Front obstacle detection and cabin safety alert system", International Journal of Computer Science \& Information Technology, vol.4, No.2, April 2012.

[2] V.Ramya, B.Palaniappan, K.Karthick and Subash Prasad "Embedded System for vehicle cabin toxic gas detection and alerting”, Journal of Elsevier Procedia Engineering, 30(2012).

[3] V. Ramya, B. Palaniappan, "Embedded Technology for Vehicle cabin safety Monitoring and Alerting System", International Journal of Computer Science Engineering and Applications, Volume 2-No.2, April 2012.

[4] V. Ramya and B. Palaniappan, "Embedded Home Automation for Visually Impaired", International Journal of Computer Applications, Volume 41-No. 18, March 2012.

[5] Yao-Jan Wu, Feng-Li Lian, and Tang-Hsien Chang, "Traffic Monitoring and Vehicle Tracking using
Roadside Cameras", in IEEE International Conference on Systems, Man, and Cybernetics, Taipei, Taiwan, October, pp. 4631-4636, 2006.

[6] B. L. Tseng, C. Y. Lin, and J. R. Smith, "Real-time video surveillance for traffic monitoring using virtual line analysis," in Proc. IEEE International Conference on Multimedia and Expo, Lausanne, Switzerland, vol. 2, August pp. 541-544, 2002.

[7] Kunfeng Wang, Zhenjiang Li, Qingming Yao, Wuling Huang and Fei- Yue Wang,"An automated vehicle counting system for traffic surveillance", IEEE International Conference on Vehicular Electronics and Safety, Dec. 2007.

[8] V. Kastrinaki, M. Zervakis, and K. Kalaitzakis, "A survey of video processing techniques for traffic applications," Image Vis. Comput., vol. 21, no. 4, pp. 359-381, Apr. 2003.

[9] W.-L. Hsu, H.-Y, M Liao, B.-S. Jeng, and K.-C. Fan,"Real-time traffic parameter extraction using entropy," Proc. Inst. Elect. Eng.-Vis. Image Signal Process, vol. 151, no. 3, pp. 194-202, Jun. 2004.

[10] K.Kiratiratanapruk,P.Dubey,S.Siddhichai,“Agradientbased foreground detection technique for object tracking in a traffic monitoring system", IEEE International Conference on Advanced Video and Signal-Based Surveillance, Como, Italy, September, pp.377- 381, 2005.

[11] K. Kiratiratanapruk and S. Siddhichai, "Vehicle Detection and Tracking for Traffic Monitoring System", IEEE Region 10 Conference, Hong Kong Convention and Exhibition Centre, Wan Chai, Hong Kong, November 14-17, 2006.

[12] Kim-Sung Jie and Ming Liu, "Computer Vision based Real-Time Information Acquisition for Transport Traffic", In Proc. of IEEE International Conference on Information Acquisition, Hong Kong and Macau, China, July, pp.164-169, 2005.

[13] R. Cucchiara, M. Piccardi, and P. Mello, "Image analysis and rule-based reasoning for a traffic monitoring system,” IEEE Trans. Intell. Transp. Syst., vol. 1, no. 2, pp. 119-130, Jun. 2000.

[14] C. Stauffer and W. E. L. Grimson, "Adaptive background mixture models for real-time tracking," in Proc. IEEE Conf. Comput. Vis. Pattern Recog., Jun. 1999, pp. 246252.

[15] J. Kong, Y. Zheng, Y. Lu, and B. Zhang, "A novel background extraction and updating algorithm for vehicle detection and tracking," in Proc. IEEE Int. Conf. Fuzz. Syst. Knowl. Discovery, 2007, pp. 464-468.

[16] Kan, C., Ervin, R., Underwood, S., Rizzoni, G., Red, T., "Planning Study for a Michigan-Based Program in Intelligent Vehicle-Highway Systems," The University of Michigan, College of Engineering, Transportation Research Institute, Ann Arbor, MI, May 2000.

[17] Kenue, S.K., "LaneLok: Detection of Lane Boundaries and Vehicle Tracking Using Image-Processing Techniques - Part 11: Template Matching Algorithms," Vehicle Systems. 
[18] Research, General Motors Research Laboratories, GMR6503, November 2004.

[19] Hitoshi Miyata, Makoto Ohki, Yasuyuki Yokouchi, Masaaki Ohkita , "Control of the autonomous mobile robotDREAM-1 for a parallel parking," Department of Electrical and Electronic Engineering, Faculty of Engineering, Tottori University, 4-101, KoyamaMinami, Tottori 680, Japan, Mathematics and Computers in Simulation 41 (1996) 129-138.

[20] Nikolaj Zimic, Miha Mraz, "Decomposition of a complex fuzzy controller for the truck-and-trailer reverse parking problem," University of Ljubljana, Faculty of Computer and Information Science, Trzaska cesta 25, SI-
1000 Ljubljana, Slovenia, Mathematical and Computer Modelling 43 (2006) 632-645.

[21] Andrei Gurtov, Mati Passoja, Olli Aafto and Mika Raitola, "Multi- Layer Protocol Tracing in a GPRS Network," IEEE Fall VTC,vol.18, Feb. 2002, pp.55-62.

[22] Zhang Qishan, Wu Jinpei, Yang Dongkai, "Intelligent Vehicle Location and Navigation System and Apllication, BeiJing: "Science Press, 2002298.

[23] C. Demonceaux, A. Potelle, and D. Kachi-Akkouche, "Obstacle Detection in a Road Scene Based on Motion Analysis," IEEE Trans. Vehicular Technology, vol. 53, no. 6, pp. 1649-1656, Nov. 2004. 1260. 